\title{
Téoros
}

Revue de recherche en tourisme

\section{Tourisme et solidarités en Afrique}

Humaniser la modernisation pour valoriser la tradition

\section{Mimoun Hillali}

Volume 26, numéro 3, automne 2007

Tourisme et solidarité

URI : https://id.erudit.org/iderudit/1071004ar

DOI : https://doi.org/10.7202/1071004ar

Aller au sommaire du numéro

Éditeur(s)

Université du Québec à Montréal

ISSN

0712-8657 (imprimé)

1923-2705 (numérique)

Découvrir la revue

Citer cet article

Hillali, M. (2007). Tourisme et solidarités en Afrique : humaniser la modernisation pour valoriser la tradition. Téoros, 26(3), 31-37.

https://doi.org/10.7202/1071004ar d'utilisation que vous pouvez consulter en ligne.

https://apropos.erudit.org/fr/usagers/politique-dutilisation/ 


\section{Humaniser la modernisation pour valoriser la tradition}

\section{Mimoun Hillali}

Du tourisme "moteur de développement », conseillé aux pays sous-développés au milieu du XXe siècle, au " secteur d'accompagnement ", vanté à la fin des années 1980, les experts en tourisme peinent à remettre les pendules à l'heure. Et il est significatif de préciser que l'heure est actuellement à la mondialisation. Récemment, en mettant en avant l'efficacité du tourisme dans la lutte contre la pauvreté ${ }^{1}$, ils semblent revoir à la baisse les grandes promesses prémonitoires des années 1960. L'euphorie touristique née des idéologies battantes de la guerre froide est démentie par la réalité amère et crue du Sud. Pourtant, au regard des économies africaines, globalement en souffrance ${ }^{2}$, le tourisme fait figure de secteur probant et s'en tire à bon compte ${ }^{3}$.

II n'est pas besoin d'être expert pour constater qu'en Afrique la situation sociale et économique de la majorité des pays est préoccupante; elle est même dramatique dans certaines régions. Les échecs à répétition sont monnaie courante et font que les rares succès du continent brun passent inaperçus. II n'y a à vrai dire que la permanence de l'espoir, nourricière de l'optimisme, qui constitue la raison d'être de projets cautionnés par l'utopie. Une utopie possible. Autant dire qu'on est toujours dans un "flou artistique ", de surcroît élastique, ballotté entre aspirations et désillusions.

D'une part, les responsables africains, majoritairement corrompus, accusent pêle-mêle les idées exogènes et les projets parachutés - donc incompatibles avec les réalités (diverses et multiples) du continent - d'ingérence ou de myopie. D'autre part, l'expertise internationale, généreusement rémunérée, attribue les causes de ces échecs chroniques à l'incompétence des acteurs locaux et à leur incapacité à assimiler les procédés scientifiques de la perfection, tout comme les mécanismes dialectiques de la production. En somme, la mentalité africaine serait inapte à reproduire efficacement des conceptions hautement économiques ou technologiques.

Et l'on oublie que « le savoir-faire des populations fait l'objet d'évaluations pessimistes. À titre d'exemple, on fait circuler l'idée qu'il n'y a rien à faire avec les variétés locales de cultures agricoles ou encore que les habitants des régions rurales ne sont pas assez compétents pour gérer leurs ressources naturelles » (Sogge, 2003 : 198). Et si c'était vrai?

Dans ce cas, pourquoi s'acharner, avec zèle ou arrogance, à vouloir imposer des idées de gestion ou à exporter des modèles de développement que l'incompétence locale tue dans l'œuf "avec succès»? Et quel succès! Entre-temps, des populations et des économies entières subissent des secousses et des pertes irréversibles.

Au risque d'enfoncer des portes ouvertes, n'est-ce pas à l'expert chevronné de se remettre en question? Ou bien pense-t-on que l'ingérence politique "légitimée » par la vertu du savoir scientifique se doit de persévérer et ne pas capituler devant l'ignorance ? Là aussi, la démarche est bancale. Car rien ne justifie cet acharnement insensé des coopérations classiques qui semblent s'obstiner à irriguer à grands frais des "champs de dunes »! Cette allusion se réfère à l'aide internationale injectée avec fluidité mais sans résultat en Afrique depuis des décennies.

Dans ce continent de toutes les convoitises, le tourisme n'est ni un levier économique ni un frein au développement. II constitue tout bonnement une activité sensible parmi celles qui s'affirment, en termes de recettes et d'emplois. Son évolution prouve que c'est un secteur qui croit ou qui décroit en fonction de l'offre et de la demande. Les différences observées à l'échelle du continent sont plutôt dues à la qualité des acteurs, à leur capacité à saisir les opportunités aussi bien qu'à faire face aux fluctuations du marché. Bien planifié, le tourisme rapporte; mal conçu, il piétine et finit par se désorganiser. Cela revient à dire que le tourisme se doit d'occuper (ou de réoccuper) sa vraie place au sein des vocations avérées et prometteuses, autrement dit : ni monopole ni disqualification ${ }^{4}$.

Cependant, il est moralement souhaitable d'unir tous les efforts en faveur du tourisme en Afrique (gouvernements, organisations internationales, ONG, associations et collectivités locales...) afin de militer pour un tourisme humanisé qui ne lèse personne, du visiteur à l'accueillant, en passant par les populations locales, réduites parfois au statut de figurants bénévoles.

Prenant appui sur cette belle idée qu'est le tourisme social et solidaire, la présente analyse se propose de critiquer les aides spéculatives du passé, de montrer les aspects positifs des solidarités mécaniques ${ }^{5}$, en établissant, dans la mesure du possible, un lien entre solidarité et sociabilité de l'acte touristique bien planifié. II va sans dire que la bonne gouvernance, revendiquée haut et fort pour asseoir les bases d'un développement adéquat, durable et rentable, se situe sans doute quelque part entre le socialisme chimérique du passé et le capitalisme anarchique du présent. Mais que reste-t-il aujourd'hui de l'entraide coutumière africaine et qu'en est-il de sa nouvelle version mi-moderne et mi-traditionnelle, prônée par les adeptes du tourisme solidaire? 


\section{Solidarité : arme contre la pauvreté ou moyen de survie?}

La solidarité, valeur humaine hautement éthique, a sûrement joué un rôle de premier plan dans la longue et mouvementée histoire de l'humanité. II y a lieu de supposer que la solidarité et l'hospitalité, deux notions différentes en apparence mais complémentaires par essence, ont permis à l'humanité depuis la nuit des temps de survivre à des périodes effroyables, voire apocalyptiques. En second lieu, et par déduction, on est tenté de penser que la peur serait à l'origine des solidarités instinctives ancestrales (peur des phénomènes naturels, des groupes humains puissants, de l'inconnu lors des premières migrations...). « Ce qui fait le prix du voyage, c'est la peur » (Camus, 1962, cité par Robin, $1998: 54)$.

Aussi faut-il croire que la solidarité originelle, avant l'apparition des religions ou du droit, a fait office d'un altruisme qui relève du « don de rien fait au rien» (Duvignaud, 1977), tremplin somme toute judicieux pour sceller la cohésion sociale des groupements humains unis dans et par le besoin; ces associations apprivoisées par la nécessité ont tôt compris l'intérêt vital de la vie en communautés solidaires régies par la bienséance d'un socialisme dit primitif (cueillette collective, chasse par hordes, déplacements en groupes, défenses mutuelles...). Mais qu'avons-nous hérité de ces lointaines pratiques préhistoriques et archaïques?

En fouillant dans l'histoire des solidarités primaires, il s'avère que toutes les religions du monde s'en sont inspirées par genèse. Elles y expriment l'ambition d'élever l'Homme au centre de l'existence, pour en faire un symbole accompli, à l'image de Dieu, qui reflète la perfection de la Création à laquelle aide, assistance et respect sont impérativement dus.

Toutefois, les concepts de la solidarité divergent d'une religion à l'autre et d'une croyance ou culture à une autre, alors que leurs fondements de base tendent vers un même but : aider le prochain. La fraternité, la charité et l'hospitalité, trépied déontologique de toutes les morales d'ordre spirituel, mystique ou animiste, quel que soit leur ancrage religieux céleste (monothéisme), leur perception philosophique existentielle (bouddhisme) ou leur rite socioculturel relationnel (animisme), ne sont à l'origine que des actes émancipés des solidarités humainement appréciées. Chose que l'homo eco- nomicus des temps modernes a tendance à oublier ou à négliger pour les besoins de la compétition.

De nos jours, les préoccupations saillantes ne sont plus à l'altruisme, pour cause de productivité ou de compétitivité ; la lutte pour la réussite et la course à la richesse imposent des conduites où le raisonnement matériel, censé conduire à la fortune, l'emporte sur le sentiment philanthropique prodigue, porteur d'un idéalisme social peu "compétiteur », mais intégrateur. Aujourd'hui, avec un minimum de volonté et suffisamment d'éthique, la rentabilité du secteur touristique pourrait résulter d'une combinaison réfléchie entre la solidarité mécanique, propre aux pays en développement, et la solidarité organique, œuvre des pays développés. Ce vœu fraternel projette de faire la synthèse de deux types de solidarités en balisant les voies d'un rapprochement passionnant entre cultures traditionnelles et cultures modernes sur la base d'échanges à caractère social et économique équitables.

Cependant, il n'existe pas de recettes miracles ni de "normes » ou de « labels » qui permettent de standardiser les pratiques pour garantir les fondements et les principes qui permettent au tourisme solidaire de s'épanouir sans déviation à l'échelle continentale selon des règles quantifiables, mesurables et contrôlables. Les quelques chartes élaborées pour sensibiliser les touristes et les populations d'accueil font l'objet de critiques ouvertes. Pourtant, elles n'imposent rien et ne s'imposent qu'à ceux qui y adhèrent avec conviction et volontarisme.

Plusieurs auteurs (Enriquez, 1993 ; Moussé, 1992 ; Doucet, 2004 [cités par Delisle et Jolin, 2007]) ont pourtant critiqué cette formalisation de l'éthique en n'y voyant au mieux qu'un outil de management, au pire un habillage hypocrite, un exercice de relations publiques ou une forme de perversion d'une véritable réflexion éthique (Delisle et Jolin, 2007 : 21).

À la notion de conviction s'ajoute la multiplication des visions. Aux dires des experts lors de l'élaboration de l'étude du BITS (voir l'article «Les concepts et les réalités du tourisme social et du tourisme solidaire en Afrique " dans ce numéro de Téoros), l'accent doit être mis sur les diversités innombrables et complexes du continent africain en faisant ressortir :
- la pluralité des conceptions de ce que peut être le tourisme social et le tourisme solidaire en Afrique ;

- la difficulté d'amalgamer la tradition et la modernité ;

- la prédominance des jeunes dans le continent africain et leurs attentes spécifiques en matière d'activités récréatives ;

- les contraintes liées à la pauvreté (souvent extrême) d'une majorité des populations du continent ;

- les dimensions socioculturelles de la problématique de l'accès au tourisme qui ne se résume pas aux seuls problèmes économiques des populations africaines;

- le poids de l'imaginaire et du sacré qui commandent aux rites célébrant les pratiques liées à ce type de tourisme...

Ces problèmes découlent en majorité de la persistance des antagonismes entre les héritiers des pouvoirs traditionnels et les tenants du pouvoir moderne. Cette pesanteur sociopolitique tend à « fossiliser » les élites et les dirigeants secrétés par des décennies de pouvoir sans partage.

\section{Pour en finir avec le foccartisme ${ }^{6}$ : ni ingérence politique ni assistance idéologique}

De prime abord, il est très important, lorsqu'il s'agit d'analyses comparatives ou d'écrits critiques qui mettent en relation les idées du Nord avec celles du Sud, de clarifier sobrement certains concepts qui s'avèrent être, pour diverses raisons, de faux amis. II s'agit de notions qui s'enveloppent de résonances ordinaires, mais qui n'ont pas forcément de sens universel unanimement admis, à cause des dérives médiatiques, des rigidités théologiques ou des abus politiques (cf. la notion du bien et du mal). Sans doute faudrait-il partir des actes et non des symboles, encore moins des idéologies, pour espérer franchir l'image imposante (ou imposée) des choses médiatisées pour faire tomber quelques œillères ? " À vrai dire, tout consiste en fin de compte à accepter la pluralité culturelle de l'être sans brusquer sa manière sociale d'être » (Hillali, 2003: 52).

Au-delà des «vérités absolues" ${ }^{7}$ ", inévitablement polémiques parce que marinées à la sauce doctrinale des acteurs puissants ou partisans, tout se prête à discussion et nécessite dialogue et débats ${ }^{8}$ ouverts pour des 
échanges constructifs. II en est ainsi de la richesse et de la pauvreté à l'échelle planétaire. Et pour cause: à force d'opposer systématiquement le Nord, certes aisé mais porteur de poches de pauvretés criardes, au Sud, où certains milliardaires n'ont rien à envier à ceux des pays avancés, on tombe dans l'excès. Et l'obstination tend à faire le jeu des nouveaux " séparatistes universels". II n'y a pas de conflit de civilisations; il y a juste des conflits idéologiques attisés par des acteurs qui s'abritent derrière des arguments civilisateurs ou inquisiteurs. Car la gloire de l'humanité ne saurait se réduire à une comptabilité lugubre de défaites et de victoires suivant les guerres que se livrent "bons " et «mauvais", ponctuées de succès et d'échecs économiques. II serait même dommage de consigner dans l'histoire le bilan de telles folies. De toute façon, pour l'Afrique, berceau de l'humanité, l'Histoire ne fait que commencer, n'en déplaise à ceux qui rêvent de mettre fin à la leur.

Certes, les exclusions, les inégalités et les injustices sont le lot quotidien de notre époque, qualifiée fièrement de civilisée. Le triomphe de la science et le règne de la technologie, la recherche du confort et la fièvre de la consommation ensemencent, sous l'égide de la mondialisation, des mutations profondes qui font la part belle à l'individualisme. Or, il est bien connu que solidarité et égoïsme ne font pas bon ménage. Au mieux, ils cohabitent en un sursis des plus improbables. L'égoïsme, adulé par ambition ou par imitation, incarne une nouvelle religion, dépourvue de spiritualité généreuse, et grignote lentement l'héritage séculaire des solidarités ancestrales, y compris en Afrique... on est même tenté de dire "surtout en Afrique ", continent considéré, de l'avis des observateurs, comme le plus traditionnaliste de la planète. À première vue, il en est résulté une somme d'altérations irréversibles qui touchent aux legs sociaux des relations qui animent aides et entraides entre différentes composantes sociétales. C'est la porte ouverte à tous les abus au nom du gain et, surtout, du gain facile.

Ces mutations, brutales ou sourdes, applaudies par une minorité, sont décriées par la majorité, parce qu'elles touchent aux fondements qui régissent les alliances solidaires et les pactes fructueux des sociétés africaines. En conséquence, l'entraide ancestrale en pâtit. En somme, plus un groupe humain ou un pays arrive relativement à se moderniser, et donc à émerger en prenant de l'avance sur les autres, plus il s'emploie obstinément à disqualifier ses concurrents et ses rivaux, quitte à recourir à la force (invasion, ingérence, agression...). L'envie manipour se barricader dans un bien-être nimbé d'égocentrisme semble faire perdre à certains parvenus leurs repères traditionnels.

Cependant, ce triomphe apprécié ne manque pas de poser quelques cas de conscience. Les grands écarts sociaux et économiques, qui font le bonheur des opulents et en même temps le malheur des exclus, ne sont pas toujours sans conséquences sur les relations humaines; celles-ci sont systématiquement reflétées d'une manière ou d'une autre dans le tourisme. Ce reflet latent n'est palpable que lorsque la fortune se laisse appâter par l'éclat du dépaysement valorisant des milieux démunis.

La nature du tourisme pratiqué dans une région donnée favorise des compatibilités qui célèbrent le mariage de raison entre le visiteur et son milieu d'accueil. Dans le cas contraire, le tourisme provoque des animosités qui rendent le brassage des populations malaisé ou carrément conflictuel. Dans les deux cas, l'observateur ordinaire est mal armé pour déceler les tenants et aboutissants d'une activité aux apparences trompeuses. Les aspects séduisants mais trompeurs du phénomène font que les ententes tranquilles et les mésententes larvées d'un milieu touristique classique peuvent cohabiter longtemps en ne montrant que des signes feste de consolider une position d'arrivisme

épisodiques de conflictualités passagères, jusqu'au jour où un détonateur casuel provoque l'étincelle qui met le feu aux poudres. C'est pourquoi la responsabilité de la recherche dans ce domaine est grande ${ }^{9}$.

La brouille atteint le summum lorsque la jouissance d'une situation d'aisance suggère quelque générosité circonstancielle en guise de charité inscrite d'avance en porte-à-faux avec d'éventuelles circonstances atténuantes. Élan de bonté calculée, le geste vise à solder à bas prix quelque dette morale envers les laissés-pour-compte! «Témoignage d'altruisme, de puissance et d'expertise, l'aide est offerte avec une lueur de satisfaction dans le regard. » (Sogge, 2003 : 27). Agit-on alors par culpabilité, par pitié ou par sérénité?

Les chasseurs, comme les autres experts de l'aide internationale, raffolent de ces «mécènes " qui ne demandent de comptes à personne, puisque l'acte de charité est à la fois déculpabilisation et autosatisfaction; dans ce cas, le résultat est fréquemment sans effet en amont (donateur) et en aval (récepteur), puisque le gros de la transaction est souvent engrangé par l'intermédiaire! Depuis la Seconde Guerre mondiale, la mise en place d'une « industrie» de l'aide internationale, régentée par des réseaux "foccartisés ${ }^{10}$ ", a souvent provoqué des effets contraires aux bonnes intentions de donateurs sincères.

Pourtant, tout ne tourne pas rond dans l'aide au développement. Là où elle prédomine, la fierté et l'ambition ont cédé la place à la dépendance et à la soumission.

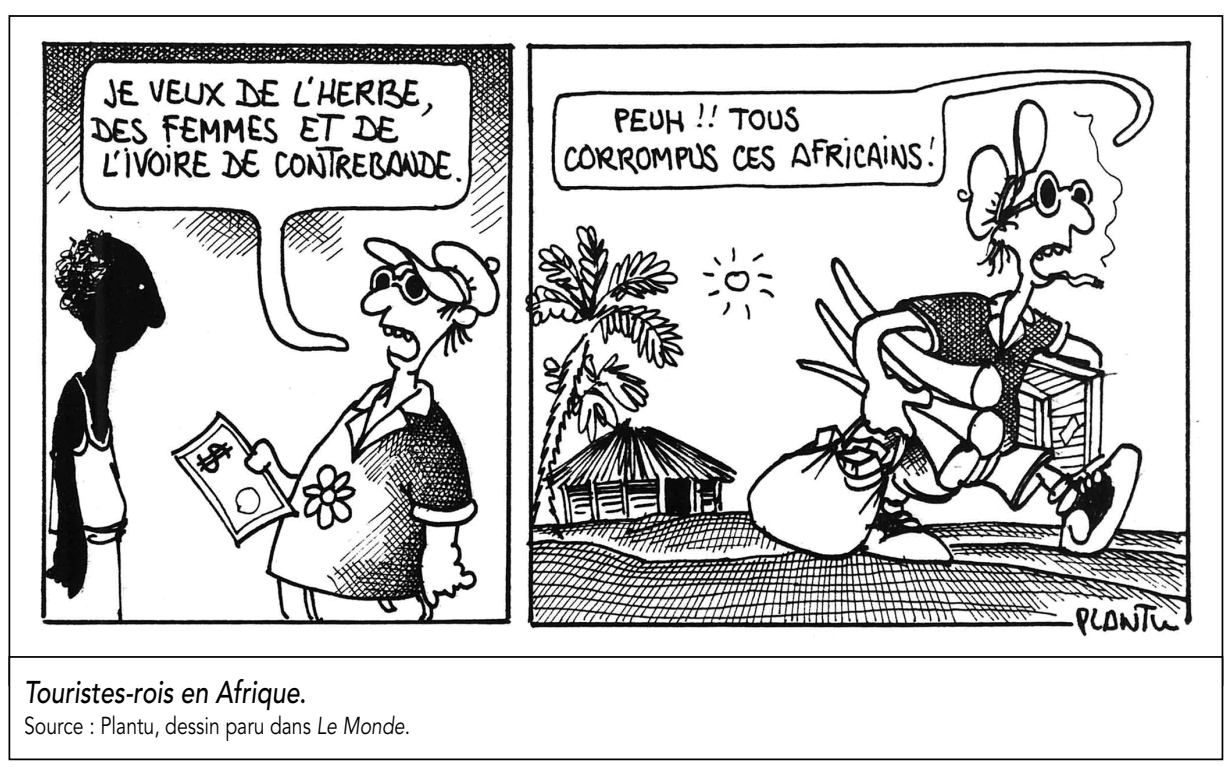


Dans certaines régions ciblées par l'aide, la gestion et les services publics se sont dégradés ou carrément effondrés, la pauvreté et l'inégalité se sont aggravées et l'insécurité règne. Les paradoxes sont parfois grotesques. Depuis 1990, l'industrie de l'aide au développement a occupé une place importante dans des sociétés qui basculent vers le crime et la violence. Au Rwanda, toutes les mains tendues pour aider ont pourtant contribué à placer ce pays au bord du génocide - tragédie suivie d'une dénégation de toute responsabilité dans son déclenchement (Sogge, 2003 : 28).

Dans pareille situation, l'aide internationale se fait de régime à régime au nom des peuples et ressemble drôlement au jeu du boomerang qui, une fois lancé, survole sa destination et revient dans la main du lanceur!

Et que dire des actions à effet d'annonce où sont «chantés » les éloges de l'assistance des «bienfaiteurs » officiels dont le but est d'accéder à davantage de notoriété ou de célébrité ? Ne faut-il pas voir dans ces situations outrageuses les germes de désobligeances larvées qui s'expriment ici et là par des ressentiments et plus tard par des violences faciles à condamner à distance, mais difficiles à apaiser sur place? À moins que ce ne soit pas là une conséquence malheureuse, mais un but recherché!

\section{Tourisme solidaire ou civisme planétaire : au nom de la dignité}

C'est pour toutes les raisons soulevées que le tourisme solidaire, dans sa forme équitable, est apprécié économiquement et convoité humainement. À ce propos, il est bon de se rappeler la sagesse du célèbre adage chinois qui déconseille l'assistance et recommande la formation ou la débrouille. II suggère d'apprendre à pêcher à celui qui sombre dans le besoin, et non à lui offrir du poisson, pour assurer son autonomie et son indépendance tout en ménageant sa dignité. II existe encore au sein de l'humanité des femmes et des hommes très sensibles aux malheurs des autres et qui sont prêts à aider, à condition de ne pas se faire berner.

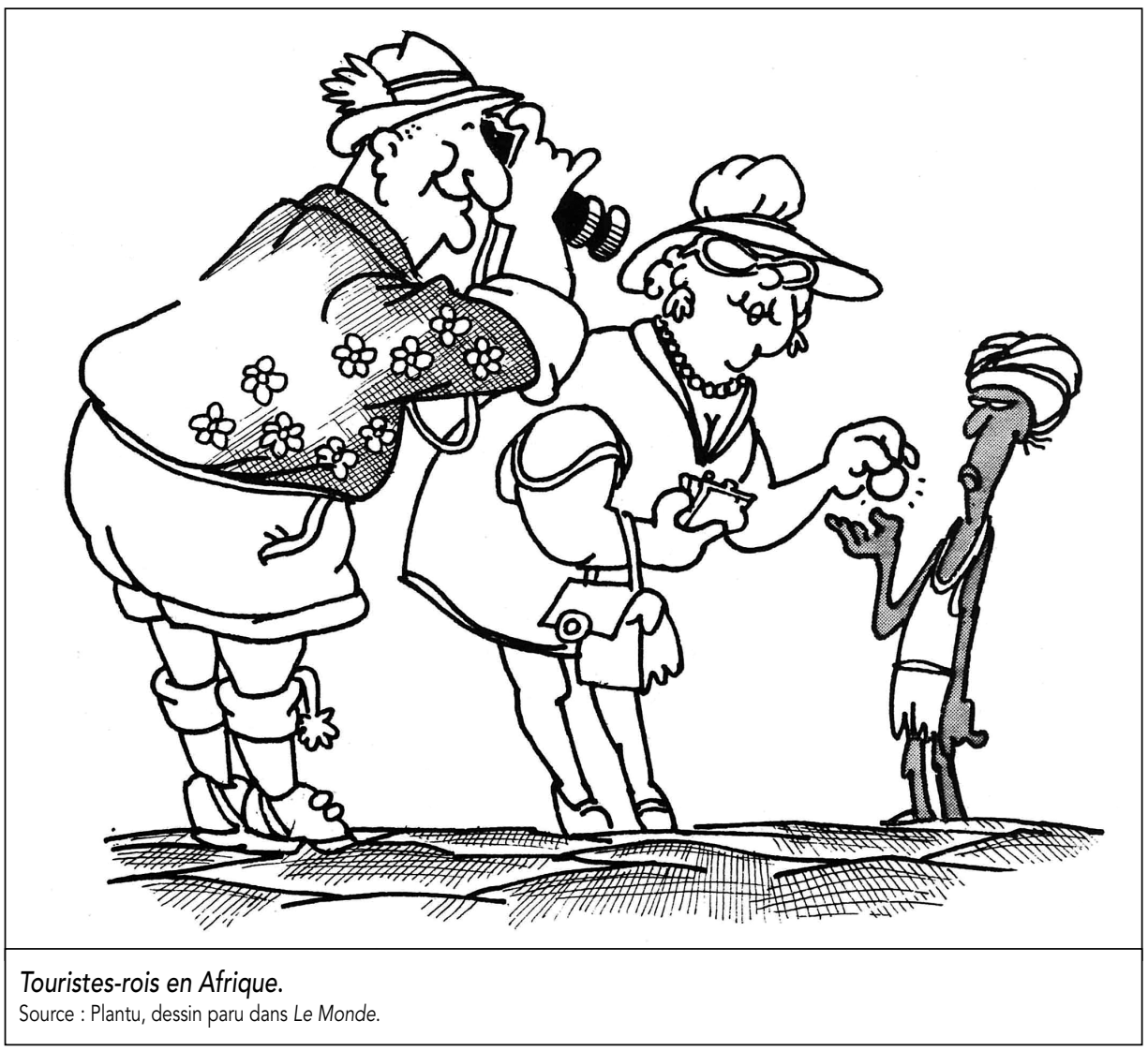
eux, de tourisme équitable et le placent au cœur des activités touristiques qui tirent leur légitimité de l'éthique.

Toutes les expériences de tourisme qui pourraient s'apparenter au tourisme équitable ont au moins les points suivants en commun :

- la population est impliquée dans l'organisation du tourisme ;

- la population est bénéficiaire financièrement des retombées de ce tourisme grâce au travail qu'elle accomplit ;

- la prestation touristique est organisée de manière telle qu'elle ne déstabilise pas les équilibres écologiques, économiques, humains et culturels locaux;

- le séjour est axé sur la compréhension des réalités locales et l'immersion culturelle (Albert et Grozelier, 2002 : 65-67).

Tourisme équitable ou solidaire, la différence des vues frôle par moments la polémique; or celle-ci n'a apparemment pas de raison d'être. Au-delà même de ces deux concepts, d'autres notions en tourisme militent pour la même cause, la bonne, bien entendu. 
Cependant, le tourisme solidaire a l'avantage de féconder les initiatives locales et les produits du terroir par l'entremise de relations amicales et commerciales qui mettent en symbiose les intérêts mutuels des visiteurs et des populations locales. Les équipements et les activités disponibles localement (hébergement, restauration, accueil, transport, animation...) sont proposés aux vacanciers, moyennant finances, par des prestataires de services formés (professionnels) et des amateurs sensibilisés (associations) du pays. Le touriste solidaire est a priori un voyageur conscient de son rôle de citoyen universel. Sensibilisé et averti, il sait voir et sentir autrement. "Le véritable voyage de découverte ne consiste pas à chercher de nouveaux paysages, mais à voir avec de nouveaux yeux ${ }^{11}$ ". Et ce nouveau regard ne fera tomber les œillères qui le briment qu'en se décidant à regarder la réalité en face.

Le contexte permet d'associer la dignité à la rentabilité ; en plus de la création des services et des emplois, de la mise en valeur des produits locaux et de la requalification des savoir-faire en déclin, une partie des revenus générés par la solidarité touristique est affectée à des systèmes collectifs durables relevant de l'intérêt général (éducation, santé, énergie douce, eau potable...) ou à des actions fédératives (fêtes de villages, initiation des jeunes, ateliers de formation...). En somme, la distribution d'une partie des recettes du tourisme solidaire se fait de façon communautaire ; elle affecte d'office, de façon directe ou indirecte, un pourcentage des revenus tou-

\section{Tableau 1}

Valeurs, acteurs, logiques d'action et dérives possibles des diverses formes de tourisme

\begin{tabular}{|c|c|}
\hline Type de tourisme & Valeurs \\
\hline Tourisme responsable & Bonne conduite, prévoyance, respect et discernement \\
\hline Tourisme social & Justice sociale, équité et égalité \\
\hline Tourisme solidaire & Partage, coopération, empathie et altruisme \\
\hline Tourisme durable & Pérennité, équité, conservation et croissance \\
\hline Tourisme communautaire & Participation, engagement, conservation, appartenance et égalité des axes \\
\hline Tourisme équitable & Justice, honnêteté, transparence et intégrité \\
\hline Tourisme intégré & Tolérance, respect, interdépendance et accueil \\
\hline
\end{tabular}

Source : Delisle et Jolin, $2007: 63$

ristiques aux populations à mobilité réduite ou à productivité insuffisante. De toute évidence, étant donné que le système n'en est encore qu'à ses débuts, il comporte des imperfections et connaît quelques ratages, lesquels sont délibérément mis en avant et attisés sourdement par ceux qui rêvent d'une mondialisation «à couteaux tirés ».

Est-il nécessaire de rappeler que les grands «monopolisateurs » du voyage, avides de la seule et unique rentabilité immédiate, mangent à tous les râteliers sans gêne ? Ils n'hésitent guère à faire du tourisme équitable ou solidaire un produit d'appel lifté par le biais d'un marketing dit «tribal », qui s'assouplit ou s'adoucit le temps d'un maquillage imposteur mais vendeur. Mais est-ce là une raison valable pour baisser les bras et laisser faire?
Dans tous les domaines socioéconomiques, le détournement des bonnes actions -et même des bonnes intentions - est toujours possible. Car le tourisme solidaire, par sa particularité de secteur de services, s'y prête, plus que le commerce équitable, par exemple, où la traçabilité des produits, grâce à leur matérialité, permet de minimiser les fraudes.

C'est d'ailleurs la raison pour laquelle la transparence doit être de rigueur. Car sans une gouvernance transparente, les slogans précurseurs ou flatteurs du solidarisme touristique, si brillants soient-ils, ne pourront aucunement éclairer les zones d'ombre où se réfugie l'intermédiaire spéculateur, voire le gentleman fraudeur, de l'aide internationale, tout comme le voyagiste extra libéral qui adopte par pur intérêt des attitudes hypocrites afin de pressurer des causes hu-

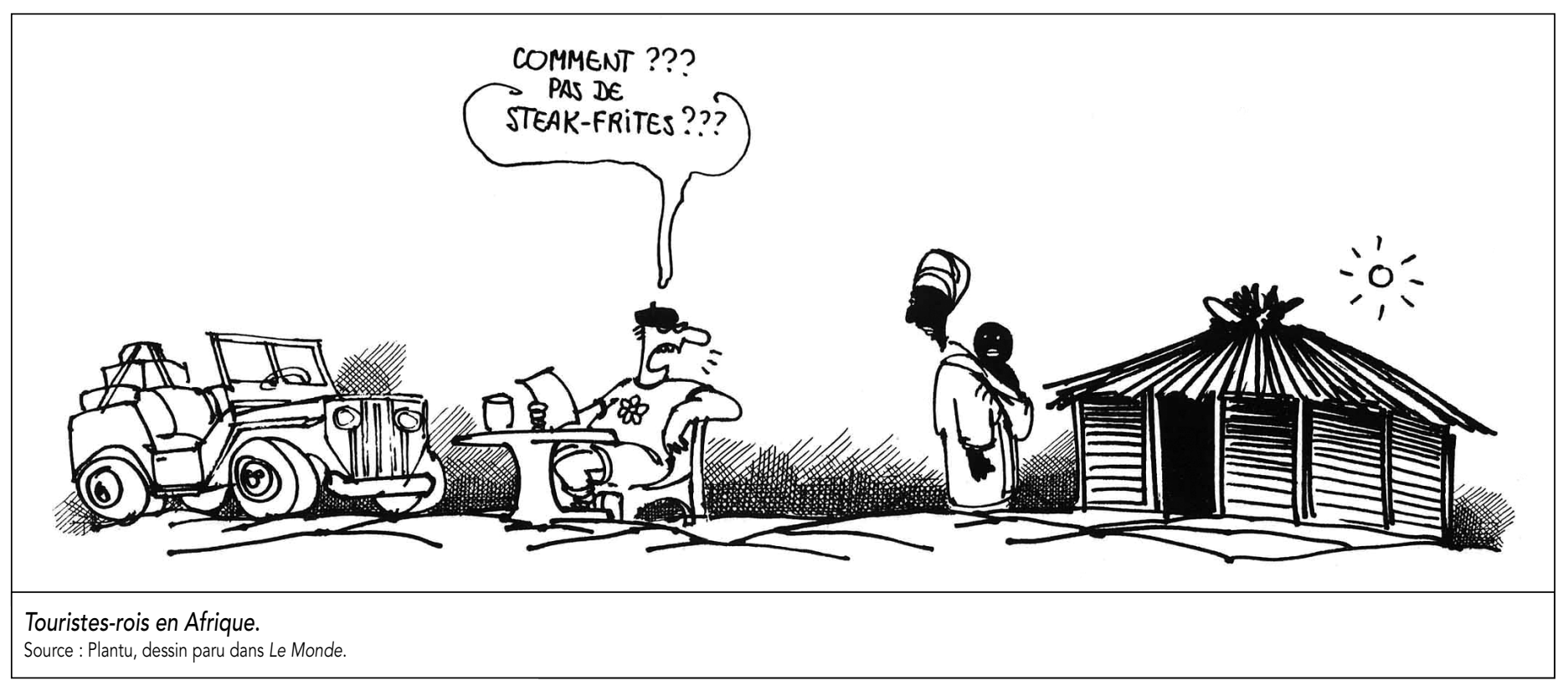


manistes rentables, qu'il méprise en principe. II faut dire que le tourisme solidaire configure un élan de rapports appropriés et de négoce équitable qui s'appuient sur des échanges planifiés proches du troc. Cet espoir de relance touristique sous-entend un mouvement altruiste, et non humanitaire, où la solidarité et la rentabilité s'allient loyalement pour mettre à l'honneur une nouvelle forme d'économie et de relation touristiques en impliquant sans distinction "droits et devoirs ». Ce jumelage des droits et des devoirs citoyens est indispensable à la renaissance du civisme, père de toutes les solidarités. II incite tout un chacun à s'y mettre avec intérêt et conviction, dans un esprit de "contribution individuelle - distribution collective ". II s'agit en fait d'un partage qui se déploie grâce à la socialisation des recettes (solidarité locale) avalisée par la collectivisation des dépenses (solidarité internationale).

De nos jours, l'Afrique constitue encore un vaste champ de solidarités mécaniques. C'est que le sous-développement constitue le terreau idéal pour l'épanouissement des groupes qui se montrent solidaires économiquement et adversaires socialement. La solidarité organique, fille de la démocratie, peine à prendre racine dans un continent où le pouvoir fort se sert d'abord, en servant ensuite ses protecteurs étrangers, véritables parrains de sa pérennité. En distribuant des miettes nationales et internationales au peuple, le donneur exige au préalable louanges et génuflexions. Sinon gare à l'embargo!

D’après E. Durkheim, les sociétés passent très progressivement d'une cohésion sociale fondée sur la solidarité mécanique à une cohésion sociale fondée sur la solidarité organique. La croissance de la population, l'amélioration des communications, etc. nécessitent et permettent la spécialisation des individus. Ces derniers nouent des liens sociaux par le biais par exemple des échanges, qui ne cessent de progresser. Un sentiment de solidarité peut donc apparaître fondé sur la reconnaissance de la complémentarité entre individus spécialisés. Ce changement social se traduit par un développement de l'individualisme; la diversité des modèles de comportement laisse une plus grande autonomie aux individus.

\section{Conclusion}

Les quelques actions expérimentées en Afrique $^{12}$ en matière de tourisme solidaire démontrent agréablement que ce type de tourisme rompt avec les systèmes d'assistance classiques, quelque peu dégradants, en mettant à l'honneur un nouveau moyen de partenariat et d'entreprenariat touristiques. De tout temps, les aides financières allouées aux pays africains en substance ${ }^{13}$, collectées dans les pays riches avec tapage médiatique, ont souvent pour but de caresser l'ego des donateurs sans produire une quelconque amélioration durable sur le terrain. Pire, il arrive parfois et par endroits que ces canaux de générosité caritative renforcent les dépendances des pays en retard. De plus, elles apportent indirectement (mais corrélativement) des soutiens regrettables aux dictatures responsables des drames et des tragédies qui servent de ferment ou d'argument à la quête des aides.

En effet nombreux sont ceux qui prétendent que l'aide elle-même ne peut être blâmée, qu'elle n'a pas fonctionné correctement parce qu'elle a été détournée à des fins politiques et commerciales égoïstes, qu'elle n'a pas favorisé les bons pays, les bons dirigeants et les bons secteurs, et qu'elle a été mal supervisée et prise à la légère. II semble alors qu'une réforme de l'aide s'impose, mais cela se résume en général à en offrir davantage (Sogge, 2003 : 29).

Pour autant, la solidarité mécanique n'est pas morte dans les pays développés ; sa sœur jumelle, la solidarité organique, n'est pas elle non plus absente des pays en développement. En Afrique, la solidarité mécanique contribue à atténuer les difficultés et les souffrances des démunis, mais elle tend, avec la mondialisation (apparemment mécanique dans le Sud), à s'estomper. C'est que les solidarités coutumières africaines sont d'un secours généreusement humain, insoupçonnable aux démunis, comme le sont ailleurs les systèmes de sécurité sociale. De ce fait, la solidarité mécanique a quelque chose de sensationnel et de spirituel ; car, en plus d'être geste ou symbole, elle est matériellement action de proximité et spirituellement consolation de dignité.
II est utile de rappeler que le troc, ou ce qui en subsiste encore, permet à l'entraide collective de s'exprimer en valeurs socialement utilitaires ou éthiques, contrairement aux solidarités corporatistes des sociétés modernes où la force de la monétarisation des biens et des produits a fini par déteindre sur les relations sociales ; cette entraide attribue aux gestes (services), aux connaissances (savoir-faire) et aux produits (consommation) des valeurs pécuniairement et uniquement matérielles. Alors, si l'humanisme n'est plus au centre des relations humaines, il faudra l'y réintroduire en recourant aux actions fiables et viables, sans verser dans la charité. De plus, les mutations sociales et économiques observées dans le monde se reflètent d'une manière ou d'une autre sur toutes les formes de rapports humains à l'échelle universelle. Le contexte s'y prête a priori, du fait que l'ignorance ne cesse de reculer au profit du savoir. Sinon, il va falloir se résigner à penser amèrement que les aïeux, malgré la dureté de leurs conditions de vie, possédaient un sens de la solidarité que les générations actuelles n'ont plus.

De ce fait, le tourisme solidaire est prédisposé à réhabiliter des pratiques coutumières en recourant à la communion de l'authenticité et de la modernité, dans un style hybride aux résultats prometteurs. Ce mariage de raison entre un secteur moderne et une démarche traditionnelle est capable de procurer satisfaction (visiteurs solidaires) et revenus (prestataires sociétaires) ; ce type de tourisme est en mesure d'engendrer des alliances éthiquement appréciables où le visiteur et l'accueillant se rencontrent autour des valeurs humaines communément définies, en se rendant réciproquement service. Ni vue d'en haut, ni perçue d'en bas, l'échelle sociale du tourisme solidaire est une voie aplanie par l'intérêt général mutuel.

Mimoun Hillali est professeur à l'Institut supérieur international de tourisme de Tangers, Maroc. 
Notes

1 «Jusqu'à présent, treize projets ST-EP ont leur financement approuvé, pour un montant d'environ un million USD, avec pour bénéficiaires dix-huit pays (Éthiopie, Gambie, Guinée, Honduras, Kenya, Madagascar, Mali, Mozambique, RDP Laos, Tanzanie, Vietnam, Zambie et un projet régional en Afrique occidentale). Parallèlement, vingt-cinq projets ST-EP sont en cours d'exécution par l'OMT avec des fonds de la SNV (Organisation des Pays-Bas pour le développement), pour un total d'approximativement 1,2 million d'euros et avec pour bénéficiaires l'Albanie, le Cambodge, le Cameroun, l'Éthiopie, le Monténégro, le Népal, le Niger, l'Ouganda, le Rwanda et les pays de la SADC. L'Italie finance huit projets ST-EP en Colombie, au Ghana, au Guatemala, au Nicaragua et au Mali. Le financement d'autres projets a été approuvé en vue de leur réalisation en 2007. » (OMT 2007, informations publiées sur le site : [http://www.world-tourism.org/ francais/newsroom/Releases/2007/mars/ globa_climate_f.htm].)

2 Trente-quatre pays africains font partie "des 50 pays, classés par les Nations Unies comme pays les moins avancés (PMA) compte tenu du bas niveau de leur PIB [produit intérieur brut] par habitant, de leur faible capital humain et de leur grande vulnérabilité économique". (Statistiques officielles de l'OMT, citées par le Bureau international du tourisme social (BITS, 2007 : 3) dans son étude sur l'Afrique.)

3 Voir les statistiques officielles de l'OMT, citées par Bélanger, Jolin et Mignon dans l'article précédent du présent numéro de Téoros, intitulé : "Les concepts et les réalités du tourisme social et du tourisme solidaire en Afrique ».

4 Durant la période de la guerre froide, les pays africains affiliés au Kremlin ont rejeté le tourisme en bloc, et parfois sans raison, et ceux affiliés à la Maison-Blanche l'ont adopté, souvent aveuglément.

5 La solidarité mécanique est l'œuvre des sociétés traditionnelles; elle se base sur l'identité des individus d'une même tribu ou ethnie, dont les membres se ressemblent du fait que l'individualité (et partant l'individualisme) est noyée dans la collectivité. Quant à la solidarité organique, relation propre aux pays avancés, elle repose sur la diversité dans l'unité à cause ou grâce à la division du travail : diversité des tâches et complémentarité des activités à la manière des organes d'un organisme vivant (cf. organique).

6 Jacques Koch-Foccart (1913-1997) dit que Jacques Foccart est, de son vivant, le "Monsieur Afrique", de 1960 à 1974. Homme de main du général Charles de Gaulle, puis de Georges Pompidou, il tisse des réseaux gigantesques et joue un grand rôle dans la politique étrangère africaine à partir de 1960, au point qu'on a pu dire qu'il était, après de Gaulle, l'homme le plus influent de la Ve République. II est souvent considéré comme étant l'instigateur de nombreux conspirations et coups d'État en Afrique durant les années 1960. En 1967, il joue un rôle flou dans le soutien français à la sécession du Biafra, en livrant des armes aux mercenaires. (Source: [http://fr.wikipedia.org/wiki/Jacques_Focc art].)

7 En ce début du XXI ${ }^{\circledR}$ siècle, deux vérités absolues se disputent la domination du monde : la religion et l'idéologie.

8 C'est notamment le cas de : liberté, démocratie, ingérence, mondialisation, durabilité, solidarité...

9 Citation tirée d'une conférence donnée par l'auteur en 2006 à l'université de Tarragone en Espagne sur le thème: Tourisme et culture en Méditerranée. (Rencontre organisée par l'Aumed / Àgora universitaire de la Méditerranée, à l'université Rivora i Virgili, 29 mars- $1^{\text {er }}$ avril 2006.)

10 En référence à Jacques Koch-Foccart, op. cit.

11 Citation figurant dans l'introduction à la deuxième partie de l'ouvrage: Voyager autrement, vers un tourisme responsable et solidaire (Martin, 2002 : 63).

12 L'espace ne permet pas ici d'énumérer et encore moins d'évaluer toutes les expériences du tourisme solidaire engagées au Maghreb (Maroc), en Afrique de l'Ouest (Burkina Faso) ou en Afrique australe (Madagascar)...

13 L'aide alimentaire d'urgence pour faire face aux famines résultant des périodes de sécheresse est un acte humanitaire louable, mais elle n'apporte pas de solution durable au sous-développement, ni d'ailleurs de réponse à ses causes.

\section{Bibliographie}

Albert, Odile, et Bernard Grozelier (2002), « Peuton appliquer les principes du commerce équitable à l'industrie touristique? ", dans Boris Martin (dir.), Voyager autrement, vers un tourisme responsable et solidaire, Paris, Éditions Charles Léopold Mayer, coll. «Dossier pour un débat ».

Bureau international du tourisme social (BITS) (2007), Étude sur les concepts et réalités sur le tourisme social et solidaire en Afrique, Bruxelles.

Bugnicourt, Jacques, et Isidore Mbaye Dieng, (Dessins de Plantu) (1982), Touristes-Rois en Afrique, Paris, Éditions KHARTALA - Enda.

Delisle, Marie-Andrée, et Louis Jolin (2007), Un autre tourisme est-il possible?, Québec, Presses de l'Université du Québec.

Doucet, Hubert (2004), "L'histoire d'une confiscation ", Relations, n 690, février, p. 12-17.

Durkheim, Émile (1991), De la division du travail social, Presses de l'Université de France.

Duvignaud, Jean (1977), Le don de rien, Paris, coll. «Monde ouvert », Stock.

Enriquez, Eugène, (1993), "Les enjeux éthiques dans les organisations modernes", Sociologie et sociétés, vol. 25, n¹, p. 25-38.

Janowitz, Morris (1980), "Observations on the Sociology of Citizenship: Obligations and Rights ", Social Forces, vol. 59, n 1, cité par Rainer Zoll dans [www.erudit.org/revue/ socsoc/1998/].

Hillali, Mimoun (2003), Le tourisme international vu du Sud, essai sur la problématique du tourisme dans les pays en développement, Québec, Presses de l'université du Québec.

Martin, Boris (2002), Voyager autrement, vers un tourisme responsable et solidaire, Paris, Charles Léopold Mayer.

Moussé, Jean, (1992), "Le chemin de l'éthique », Revue française de gestion, mars-avril-mai.

Robin, Christian (1998), Paroles de voyageurs, Albin Michel.

Sogge, David (2003), Les mirages de l'aide internationale (quand le calcul l'emporte sur la solidarité), Tunis, coll. « Enjeux Planète », Cérès Productions. 